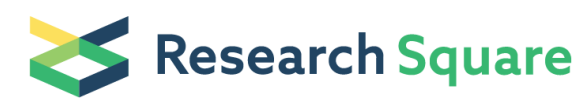

Preprints are preliminary reports that have not undergone peer review.

They should not be considered conclusive, used to inform clinical practice, or referenced by the media as validated information.

\section{Decline in influenza cases in Mexico after the implementation of public health measures for COVID- 19}

\section{Arellanos-Soto Daniel}

Department of Biochemistry and Molecular Medicine. School of Medicine, Autonomous University of Nuevo Leon https://orcid.org/0000-0002-2707-9754

\section{Padilla-Rivas Gerardo}

Department of Biochemistry and Molecular Medicine. School of Medicine, Autonomous University of Nuevo Leon https://orcid.org/0000-0003-3243-8843

\section{Ramos-Jimenez Javier}

Department of Internal Medicine. School of Medicine, Autonomous University of Nuevo Leon https://orcid.org/0000-0002-4701-7972

\section{Galan-Huerta Kame}

Department of Biochemistry and Molecular Medicine. School of Medicine, Autonomous University of Nuevo Leon https://orcid.org/0000-0002-3495-0528

\section{Lozano-Sepulveda Sonia}

Department of Biochemistry and Molecular Medicine. School of Medicine, Autonomous University of Nuevo Leon https://orcid.org/0000-0002-6690-7428

\section{Martinez-Acuña Natalia}

Department of Biochemistry and Molecular Medicine. School of Medicine, Autonomous University of Nuevo Leon https://orcid.org/0000-0001-7147-9635

\section{Treviño-Garza Consuelo}

Department of Pediatrics, Hospital Universitario "Dr. Jose E. Gonzalez", Autonomous University of Nuevo Leon, Secretariat of Health of Nuevo Leon state https://orcid.org/0000-0002-6544-3780

\section{De la O-Cavazos Manuel}

Department of Pediatrics, Hospital Universitario "Dr. Jose E. Gonzalez", Autonomous University of Nuevo Leon, Secretariat of Health of Nuevo Leon state https://orcid.org/0000-0002-8123-1294

\section{Montes de Oca-Luna Roberto}

Department of Histology, Hospital Universitario "Dr. Jose E. Gonzalez", Autonomous University of Nuevo Leon, Secretariat of Health of Nuevo Leon state https://orcid.org/0000-0001-8253-680X

\section{Rivas-Estilla Ana Maria ( $\square$ amrivas1@yahoo.ca )}

Department of Biochemistry and Molecular Medicine. School of Medicine, Autonomous University of Nuevo Leon https://orcid.org/0000-0003-2311-2534

\section{Short Report}


Keywords: SARS-CoV-2, Influenza, COVID-19, Epidemiological (EPI) week

Posted Date: November 24th, 2020

DOI: https://doi.org/10.21203/rs.3.rs-113558/v1

License: @ (i) This work is licensed under a Creative Commons Attribution 4.0 International License. Read Full License 


\section{Abstract}

Mexico took swift action and has strictly followed mitigation measures to prevent the spread of coronavirus disease, COVID-19. The present study compared indicators of influenza activity in 2020 before and after public health measures were taken to reduce COVID-19 with the corresponding indicators from three preceding years and the immediate one, and the potential decrease in seasonal influenza cases. Nationwide surveillance data revealed a drastic decline in influenza diagnosis in outpatient clinics and public hospitals, positivity rates of clinical specimens, and confirmed severe cases during the following 10 weeks of 2020 as lockdown activities and control measures were established compared with the same period of 2019, suggesting that the measures taken for COVID-19 were effective in reducing the spread of other viral respiratory diseases in our country.

\section{Introduction}

Public health measures, including public education and physical distancing, were implemented in Mexico to reduce transmission of COVID-19 after the first few cases were reported in epidemiological week 3 (January $13,2020)$ and public awareness was increased. ${ }^{1}$ We examined the effect of these COVID-19 measures on influenza incidence as a proxy for determining the overall potential reduction in respiratory virus transmission.

Mexico, like other Latin American countries, established population confinement and a reduction in its economic activity to face the coronavirus pandemic and the respiratory disease it generates, COVID-19. The Mexican Ministry of Health established a package of prevention measures called "National Protocol for Healthy Distance", effective as of Monday, March 23, 2020, to prevent the spread of COVID-19 in the community. ${ }^{2}$ These control strategies focused on social distancing and basic prevention measures and were aligned with those used for other common respiratory viral infections such as influenza: use of face masks, avoiding contact when greeting, hand washing, cough etiquette, temperature monitoring, home confinement, and avoiding close contact with sick individuals. In addition to this, cancellation of large-scale events, the lockdown of activities at all levels of education and workplaces (e.g., segregated teams and home-office working wherever possible) and non-essential activities of the public, social, and private sectors were temporarily suspended, and the reprogramming of massive events was raised. Also, intensive public health education on public and personal hygiene and social responsibility was established. ${ }^{3}$

We compared influenza activity indicators in 2020 before and after public health measures were taken to reduce COVID-19 with the corresponding indicators from the three preceding years and the immediately previous one, as well as the involvement in the decrease of seasonal influenza cases.

\section{Methods}

We obtained routine sentinel surveillance data on influenza-like illnesses (ILI) from the national network of the National Health Secretariat and the National Centre of epidemiological surveillance for all weeks since 
$2016 .{ }^{4} \mathrm{ILI}$ were defined as fever $\left(>38^{\circ} \mathrm{C}\right)$ and cough. ILI samples were tested and registered per week, and the percentage of influenza positivity was determined.

We evaluated whether the influenza trends in the 2019-2020 season were different before and after the COVID-19 National Protocol for Healthy Distance was implemented compared to previous seasons by using a quasi-experimental difference-in-difference design. We extracted weekly reports of seasonal influenza data from the 2016-17 season to the 2019-20 season from epidemiological (EPI) week 40 to EPI week 30, using the open-access databases of the public health authority. ${ }^{4}$

We compared the number of influenza cases in season 2019-2020 and the average of the corresponding periods in the previous three influenza seasons (2016-2019). T-tests were performed for each EPI week using IBM SPSS Statistics for Windows, Version 23.0. ${ }^{\mathbf{5}}$

\section{Results}

In Mexico, influenza incidence follows a yearly bimodal pattern: the influenza season and the inter-seasonal period. The influenza season spans from October to March, including autumn and winter (two years, weeks 40 to 20 ).

We compared indicators of influenza transmissibility (confirmed cases and deaths) in 2020 against the average from corresponding periods in the three preceding influenza seasons (2016-2020). Figure 1 shows trends in seasonal influenza cases from the 2016-2017 season to the 2019-2020 season in Mexico. The average influenza activity peaked in EPI weeks 3-7 in the last four influenza seasons and declined by week 15 every year (Fig. 1). Annually in our country, on average, around 500-1000 people die from laboratoryconfirmed influenza (Fig. 1). ${ }^{6}$

In Mexico, the number of seasonal influenza cases in the 2019-20 season was lower after COVID-19 transmission compared to previous years (Fig. 1 and 2A). We observed the peak of influenza cases in week 4. Particularly, the average of influenza cases at week 4 was significantly different $(P<0.05)$ from the previous two influenza seasons (587 vs $357.33 \pm 99.6$; respectively).

The number of influenza cases decreased below the average of previous years in EPI week 12 (2017-2019). In addition, we observed that after EPI week 12, in Mexico, 9 weeks had passed since the detection of the first SARS-CoV-2 case and the number of COVID-19 cases was increasing (Fig. 2A). The healthy distance protocol was officially established in EPI week 13. It was observed that simultaneously the cases of influenza were decreasing and were unusually low after week 20 compared to previous years. Even when measures to contain the transmission of the new respiratory disease were established (EPI week 13), the number of COVID-19 cases increased while prevalence of influenza declined (Fig. 2A). In fact, the number of influenza cases in 2020 decreased significantly $(P<0.05)$ from EPI week 22 to 30 compared to the seasons 2016-2019 (Fig. 2B). It is important to highlight that this phenomenon indicates a significant difference in the transmission mechanisms and the pathogenesis of these two diseases. 
We performed a weekly paired difference $t$-test using IBM SPSS Statistics for Windows, (Version 23.0). ${ }^{\mathbf{5}}$ We found that the percentage of influenza cases decreased by $64 \%(p=0.001)$ and the estimated daily number of influenza cases decreased by $76 \%(p=0.002)$ in EPI week 20 of season 2019-2020 compared with the preceding years (Fig. 2B). Additionally, before EPI week 20, the number of deaths in 2020 from influenza per EPI week was statistically similar ( $P>0.05)$ to the average of the seasons 2016-2019 (Fig. 1 and 2C). In contrast, we saw no significant changes in deaths, only in the percentage of influenza positivity $(31 \% ; p=$ 0.008), in EPI weeks 21 to 30 of 2019-2020 compared with preceding seasons (Fig. 1 and 2C).

The COVID-19 epidemic has altered social and health behaviors, resulting in an unexpected reduction of seasonal influenza cases. Influenza disease has not been detected by our national laboratories since May 8th. This dramatic decrease is unlikely to be related to climatic variations as the number of laboratoryconfirmed influenza A were similar during the same weeks in 2019 (relative risk, 1.3; 95\% $\mathrm{Cl}, 0.4-3.8, \mathrm{P}=0.7$ ), which was consistent with the typical transmission pattern in Mexico.

In addition, both the number of influenza strains isolated from clinical specimens in commissioned laboratories and the positivity rate dropped drastically in 2020; the trends were different from 2019 ( $p<0.05$ for both). In the 2019-2020 influenza season (weeks 40 to 30), circulation of influenza A (H1N1) pdm09 (47\%), B (30\%), A (H3N2) (19\%) and influenza A non-subtypeable (4\%) was observed. In week 20, the cumulative positivity rate was $8.9 \%$, which decreased by week 30 with only two cases of non-subtypeable Influenza A identified (Figure 3).

\section{Discussion}

According to the WHO, the lethality of COVID-19 is higher than for seasonal influenza (usually well below $0.1 \%)$. COVID-19 mortality seems to differ according to the region. ${ }^{6}$ Until now, it is estimated that the crude mortality ratio (the number of reported deaths divided by the reported cases) is between 3-4\%; therefore, the infection mortality rate (the number of reported deaths divided by the number of infections) will be lower. However, for both diseases, mortality is determined to a large extent by regional access to and quality of healthcare services.

In our country, the functional healthcare and surveillance systems installed, the government's efforts to identify ILI cases during the COVID-19 pandemic, and adequate laboratory capacity guarantee an appropriate influenza testing and reporting of results. As reported by other countries, public health initiatives to control COVID-19 spread probably helped to decrease influenza transmission and number of cases in May 2020 because both viruses share the same transmission mechanisms through the respiratory route and contact, although with different efficiency giving a basic reproduction number $\left(R_{0}\right)$ for COVID-19 higher than that of seasonal influenza, then a reduction in transmission could reduce the impact of COVID-19, thereby preventing the increase of mortality. The modeling of the effective reproduction number for COVID19 in Mexico in May 2020 (National Secretariat of Health) at 0.5-1, is lower (55-77\% less) than the mean estimated $\mathrm{R}_{0}$ for this virus (2.2). ${ }^{7}$ The observed $61 \%$ reduction in influenza transmission is consistent with the information above. ${ }^{8}$ 
Due to the measures implemented in our country to contain the COVID-19 cases, there were severe changes in the availability of medical care and assistance, including hospitalization. Avoidance of medical care during this period may be a major confounder in interpreting our results. It is important to note that due to the similarity in symptoms between COVID-19 and influenza and the low number of COVID-19 patients in Mexico ( $<200$ cases as of March 21, 2020), ILI patients would seek help for a differential diagnosis. The reduction of the availability of medical care also did not explain the lower number of severe influenza cases seen in 2020 (Figure 2C). Therefore, we believe that the decline in influenza activity in Mexico in 2020 is the result of the strict control measures that were put in place in response to COVID-19.

During the last winter season, European countries and the North American region (United States, Canada and Northern Mexico) have reported fluctuations in the number of cases of seasonal influenza different from experienced in previous seasons (since EPI week 4, 2019-20 season) European countries and North America region (United States, Canada and North Mexico) have reported similar trends in the fluctuation of seasonal influenza cases from those experienced in previous seasons. In contrast, some Asian regions experienced different trends in influenza incidence during this season, from those experienced in past years. 9,10 The total number of patients infected with influenza in countries like Hong Kong, Japan, and Taiwan was significantly lower after EPI week 4 of the 2019-20 season than in previous influenza seasons. ${ }^{11}$

We can mention some limitations in our study. First, due to the seasonal bimodal pattern of influenza incidence in Mexico, it is expected that influenza cases diminish around March-May. However, this year the number of cases fell to almost zero. Second, it is important to consider an underdiagnosis of ILI's due to the fear of leaving home in search of medical attention and catching coronavirus, as well as being classified as COVID-19, all this contributes to generating an altered pattern ${ }^{11}$ Third, if we consider a similar dynamic for COVID-19, we will also have to record a higher number of cases for COVID-19 due to the underdiagnosis and the presentation of asymptomatic cases. ${ }^{12,13}$

In conclusion, we found a marked decline in influenza cases in Mexico after the implementation of public health measures for COVID-19. Definitively, the containment measures to reduce the risk of contagion for the new respiratory disease was additionally efficient for influenza, but with greater magnitude. This is partly explained by the different transmission mechanisms of these two respiratory diseases, the virulence of each viral agent, the absence of immunity in the population, and the diverse virological factors inherent in each pathogen. Our results suggest that such measures are useful in reducing the spread of viral respiratory diseases and their establishment mitigate the impact of the COVID-19 pandemic, especially now that we are entering into a new influenza season.

\section{Declarations}

\section{Author contributions}

Daniel Arellanos-Soto and Gerardo Padilla-Rivas conceived and designed the study, acquired data, and made the analysis and interpretation of data, and together with Ana María Rivas-Estilla drafted the 
paper. Daniel Arellanos-Soto made the figures. Javier Ramos-jimenez, Kame Galán-Huerta, Sonia LozanoSepulveda, Natalia Martínez-Acuña, Consuelo Treviño-Garza, Manuel de la O-Cavazos and Roberto Montesde-Oca-Luna revised it critically for important intellectual content. Ana María Rivas-Estilla gave the final approval of the version to be submitted.

\section{Additional information}

Funding: The authors declare that the research was conducted in the absence of any commercial or financial relationships that could be construed as a potential conflict of interest.

Conflicts of interest/Competing interests: The authors declare there are not conflicts of interest.

Ethics approval: Not necessary, the data analyzed is publically available on Secretariat of Health of México databases.

Consent to participate: Not necessary. We analyzed data publically available on Secretariat of Health of México databases.

Consent for publication: All the authors give their consent for this work publication. This work has not been published previously), and it is not under consideration for publication elsewhere,

Availability of data and material (data transparency): Data analyzed in this work is publically available.

\section{References}

1. COVID-19 reports. Secretariat of Health Mexico. https://coronavirus.gob.mx

2. Jornada de Sana distancia. National Health Secretariat of México. 2020. https://www.gob.mx/cms/uploads/attachment/file/541687/Jornada_Nacional_de_Sana_Distancia.pdf

3. World Health Organization. Coronavirus disease (COVID-19) advice for the public: when and how to use masks. [cited 2020 March 30]. https://www.who.int/emergencies/diseases/novel-coronavirus2019/advicefor-public/when-andhow-to-use-masks.

4. Weekly reports for Influenza epidemiological Surveillance. National Health Secretariat of México. General Direction of Epidemiology. https://www.gob.mx/salud/documentos/informes-semanales-parala-vigilancia-epidemiologica-de-influenza-2020.

5. IBM Corp. Released 2015. IBM SPSS Statistics for Windows, Version 23.0. Armonk, NY: IBM Corp.

6. World Health Organization. Influenza update https://www.who.int/influenza/surveillance_monitoring/updates/latest_update_GIP_surveillance/en/ 03 August 2020

7. Brian J Coburn, Bradley G Wagner, Sally Blower. Modeling influenza epidemics and pandemics: insights into the future of swine flu (H1N1) MC Med 2009 Jun 22;7:30. doi: 10.1186/1741-7015-7-30.

8. Soo RJJ, Chiew CJ, Ma S, Pung R, Lee V. Decreased influenza incidence under COVID-19 control measures, Singapore. [published 
online ahead of print April 27, 2020]. Emerg Infect Dis. 2020;26(8).

https://doi.org/10.3201/eid2608.201229

9. Caini S, El-Guerche Séblain C, Ciblak MA, Paget J. Epidemiology of seasonal influenza in the Middle East and North Africa regions, 2010-2016:

circulating influenza A and B viruses and spatial timing of epidemics. Influenza Other Respir Viruses. 2018;12:344-352.

10. Lee VJ, Chen MI, Yap J, Ong J, Lim W-Y, Lin RTP, et al. Comparability of different methods for estimating influenza infection rates over a single epidemic wave. Am J Epidemiol.2011;174:468-78.

https://doi.org/10.1093/aje/kwr113

11. Koo JR, Cook AR, Park M, et al. Interventions to mitigate early spread of SARS-CoV-2 in Singapore: a modelling study. Lancet Infect Dis. 2020;20:678-688.

12. World Health Organization. Q\&A: Similarities and differences-COVID-19 and influenza. Geneva: The Organization; 2020 [cited 2020 April 15]. https://www.who.int/news-room/q-a-detail/q-a-similaritiesand-differencescovid-19-and-influenza.

13. Kissler SM, Tedijanto C, Goldstein E, Grad YH, Lipsitch M. Projecting the transmission dynamics of SARS-CoV-2 through the postpandemic period. Science 2020;368:860-868.

\section{Figures}

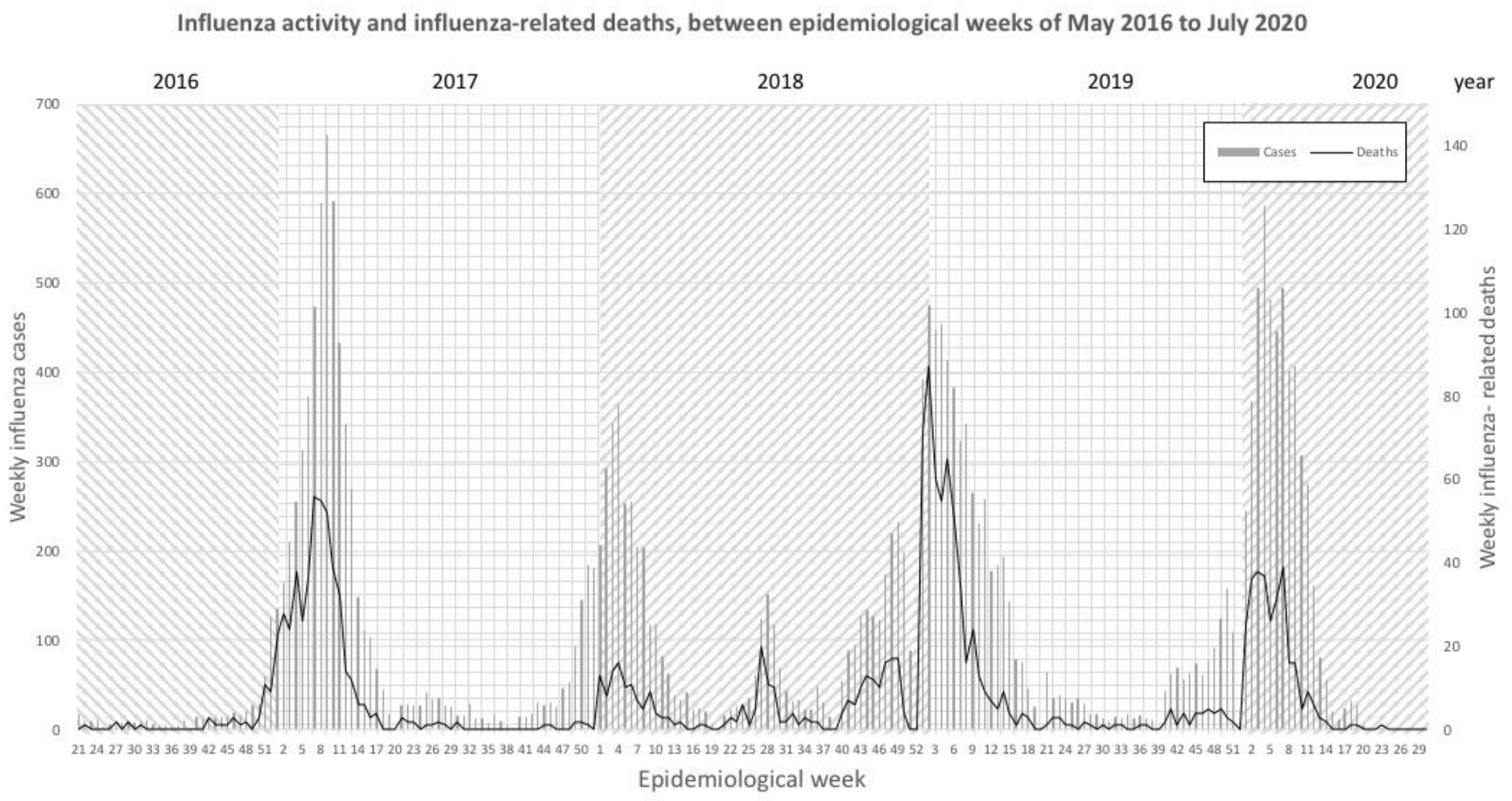




\section{Figure 1}

Trends of influenza activity and influenza-related deaths during the 2019-20 season compared with average of the preceding 3 years (between EPI weeks of May 2016 to July 2020).

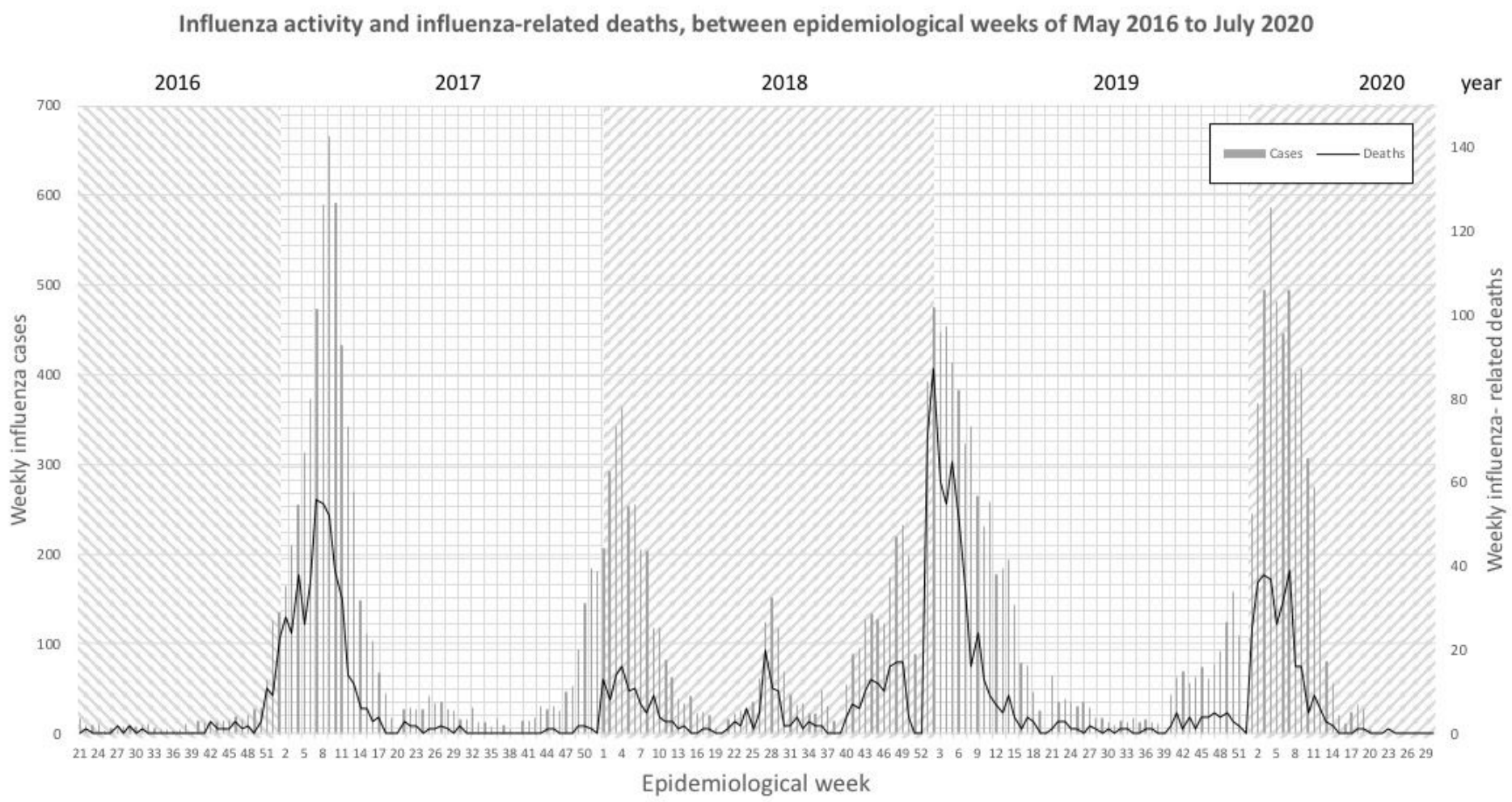

Figure 1

Trends of influenza activity and influenza-related deaths during the 2019-20 season compared with average of the preceding 3 years (between EPI weeks of May 2016 to July 2020).
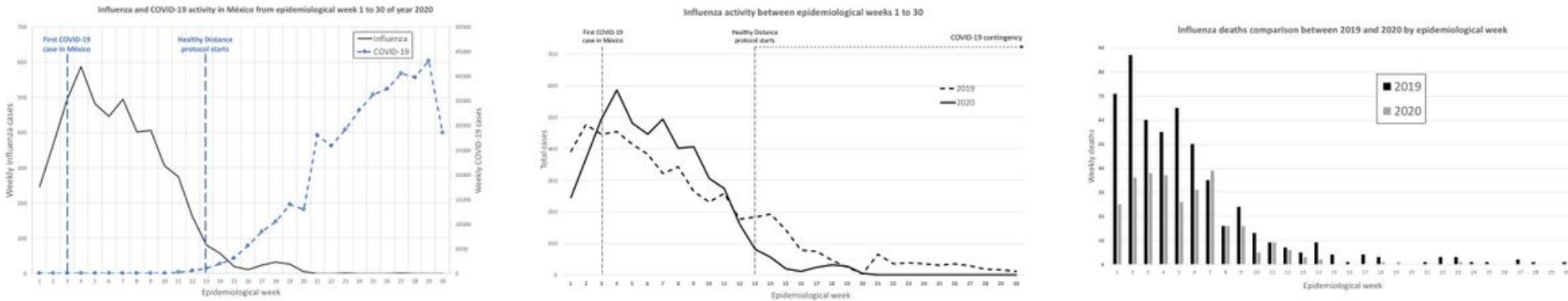

\section{Figure 2}

Trends of influenza activity during the 2019-20 season. (Left Panel) A) Influenza and COVID-19 activity in México from EPI week 1 to 30 of year 2020; (Middle Panel) B) Influenza activity between EPI weeks 1 to 30; (Right Panel) C) Influenza deaths comparison between 2019 and 2020 by EPI week. 

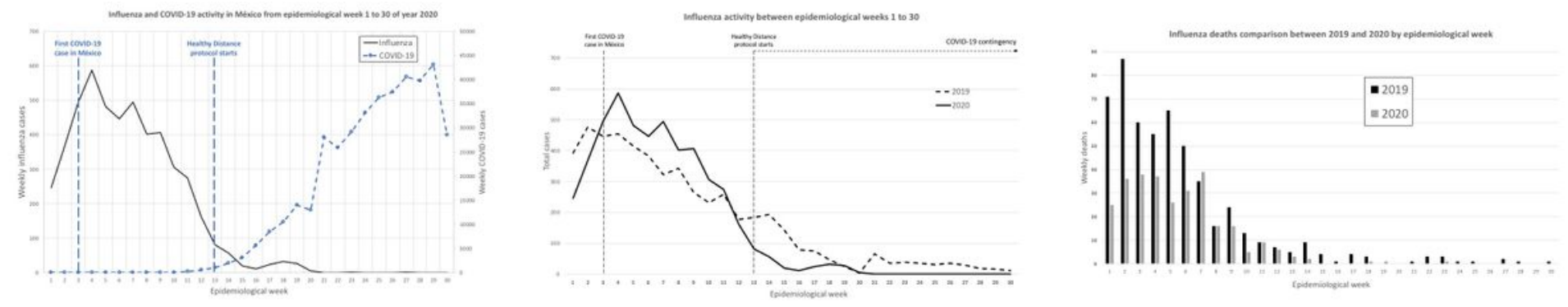

Figure 2

Trends of influenza activity during the 2019-20 season. (Left Panel) A) Influenza and COVID-19 activity in México from EPI week 1 to 30 of year 2020; (Middle Panel) B) Influenza activity between EPI weeks 1 to 30; (Right Panel) C) Influenza deaths comparison between 2019 and 2020 by EPI week.

Circulating Influenza types in epidemiological weeks 1-30 of year 2020

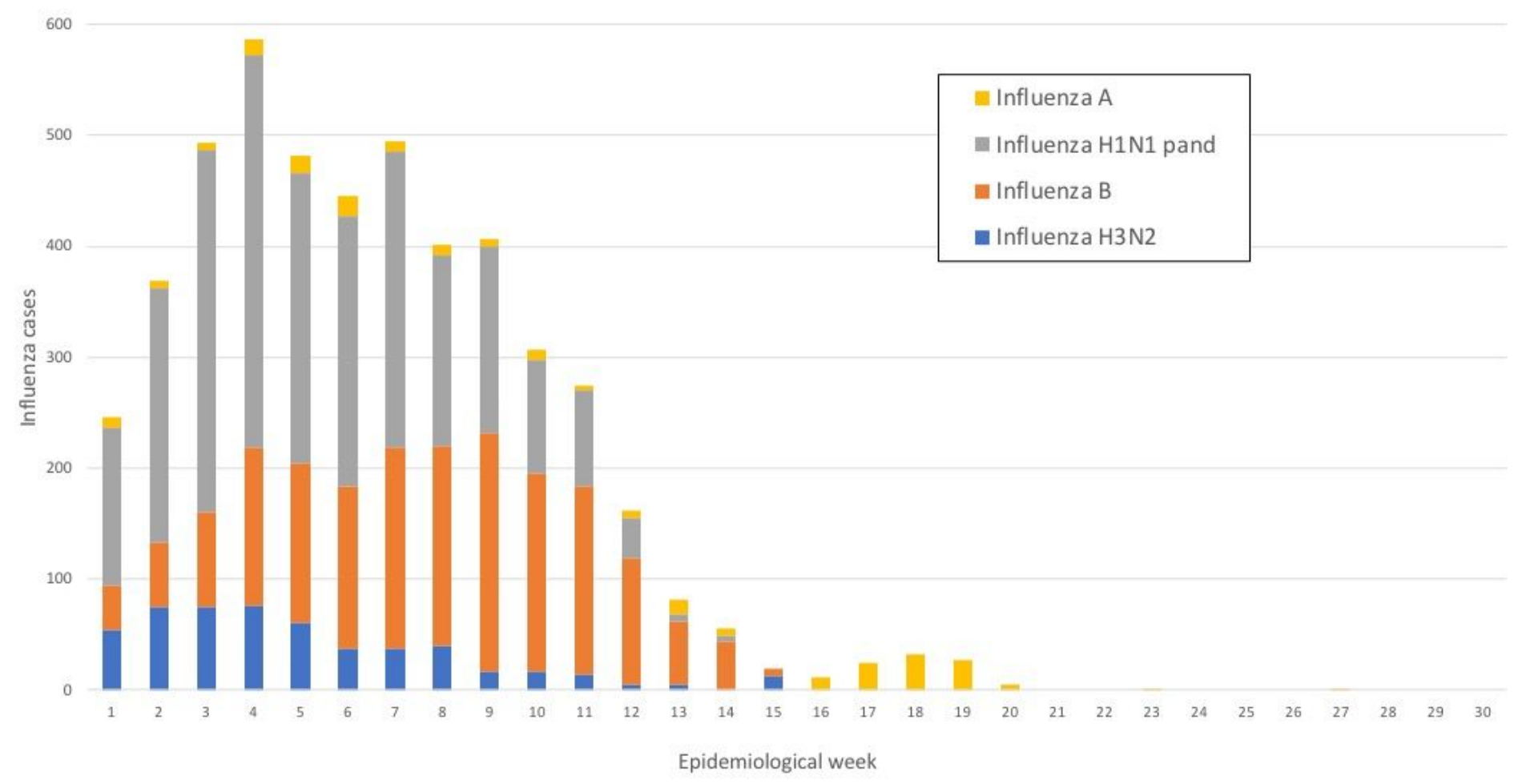

\section{Figure 3}

Circulating Influenza types in EPI weeks 1-30 of year 2020. 


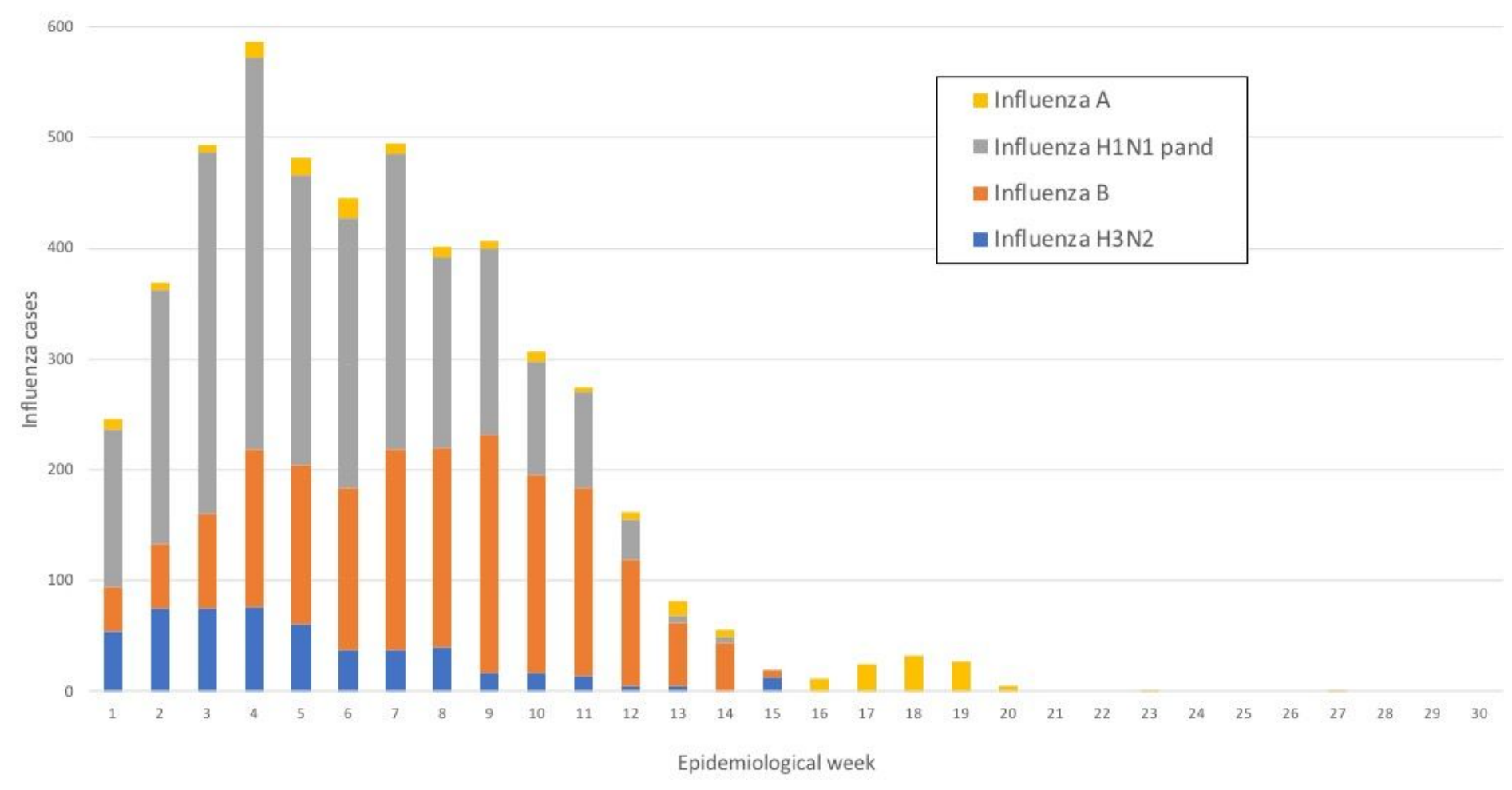

\section{Figure 3}

Circulating Influenza types in EPI weeks 1-30 of year 2020. 